\title{
Medium-energy Nuclear Physics at RHIC with sPHENIX and a sPHENIX Forward Upgrade
}

\author{
Itaru Nakagawa*† \\ RIKEN, 2-1 Hirosawa, Wako, Saitama, 351-0198 Japan \\ E-mail: itarueriken.jp
}

The preparation of the sPHENIX experiment has been underway as a next generation experiment in order to complete the research mission of the quark-gluon plasma (QGP) at the relativistic heavy ion collider (RHIC). The sPHENIX detector is a barrel type detector which covers the central rapidity region of $-1<\eta<1$ and optimized to detect jets under high multiplicity circumstances of heavy ion collisions at high energy. In addition to the QGP studies, the medium energy nuclear physics such as a hadron structure study can be pursued using the sPHENIX detector. With the high DAQ rate capability and the high hermeticity of the sPHENIX, further improvements in the precision of the gluon polarization measurements can be expected assuming a few year extension of sPHENIX running period beyond it's scheduled one 2023 - 2025. The physics opportunity can be drastically enriched if the jet detection capability is extended to the forward region during the sPHENIX running period. . The latest design of a forward detector (fsPHENIX) is composed of a high momentum resolution tracking using the magnetic field provided by a sPHENIX magnet, calorimetry and particle identification covering the pseudo-rapidity region of $1<\eta<4$. In combination with sPHENIX acceptance, the long rapidity range correlation can be studied in various collision species which is considered to give us an access to the early stage of the QGP formation. The forward region is also crucial for the spin program. Transverse spin asymmetries from Collins and Sivers effects in jets and Drell Yan yields in the unexplored large Feynman momentum $x_{\mathrm{F}}$ region, where these effects are larger, would be accessible as well as small momentum fraction gluon contribution to the proton spin $\Delta G$ ). The fsPHENIX detector is designed ultimately to be the hadron detector of the future ePHENIX in the electron ion collider (EIC) at RHIC era.

XXVI International Workshop on Deep-Inelastic Scattering and Related Subjects (DIS2018) 16-20 April 2018

Kobe, Japan

\footnotetext{
* Speaker.

${ }^{\dagger}$ for the sPHENIX Collaboration
} 


\section{INTRODUCTION of SPHENIX}

The study of Quark Gluon Plasma (QGP) is the one of the frontier of QCD studies ongoing today in the nuclear physics field. The QGP is the a new form matter, a nearly ideal strongly interacting fluid where quarks and gluons are no longer confined into nucleons. Experimentally, the QGP can be formed by the heavy ion collision at high energy. The Relativistic Heavy Ion Collider (RHIC) at Brookhaven National Lab (BNL) has had a long and successful program of the QGP study since 2000, with many upgrades that have increased the delivered luminosity considerably

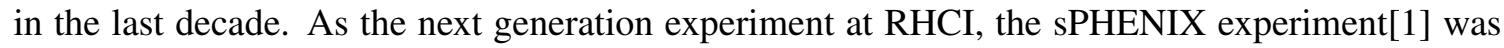
proposed as an upgrade of the PHENIX experiment. It will take advantage of the increased luminosity, and allow measurements of jets, jet correlations and Upsilons (؟s), with a kinematic reach that will overlap with measurements made at the Large Hadron Collider (LHC) as highlighted in Figure 1 . Complementary measurements at RHIC and at the LHC probe the QGP at different temperatures and densities, which are necessary to determine the temperature dependence of transport coefficients of the QGP. The sPHENIX detector will have large acceptance electromagnetic and hadronic calorimetry, as well as precision tracking, and high rate capability which are necessary for precision jet and $\Upsilon$ observables. The experiment will enable a program of systematic measurements at RHIC, with a detector capable of acquiring a large sample of events in $p+p, p+A$, and $\mathrm{A}+\mathrm{A}$ collisions.

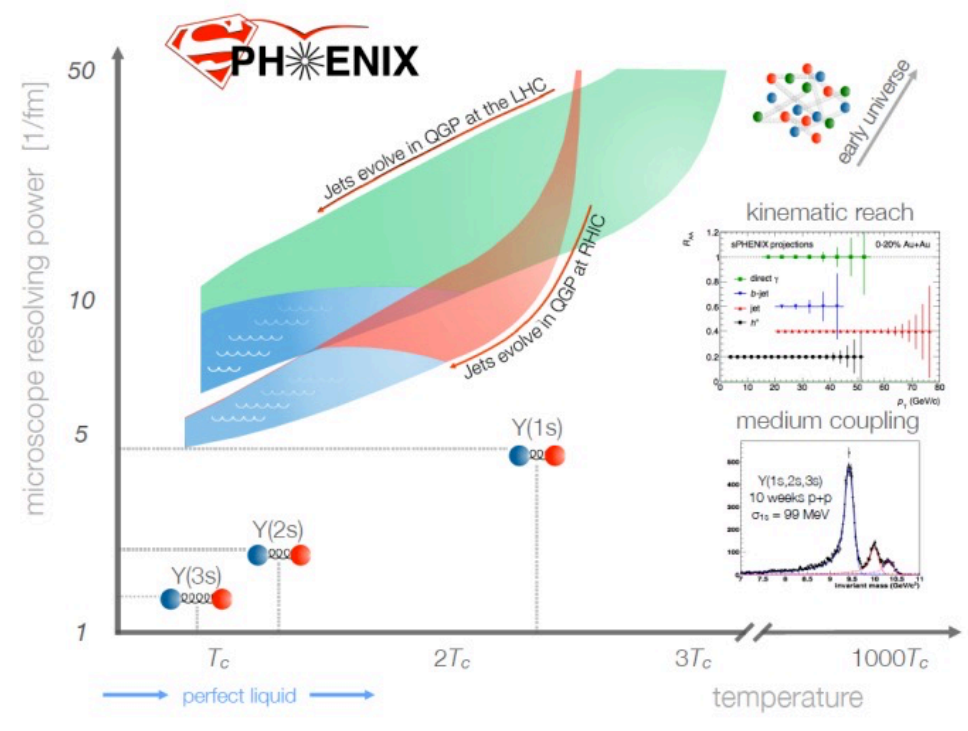

Figure 1: The microscopic resolving power and temperature of the QGP correlation. The region where jet and $\Upsilon$ measurements in sPHENIX can be explored is highlighted as well as that of LHC's.

\subsection{SPHENIX Detector}

The sPHENIX detector design as shown in Figure $\square$ is driven by the goal of measuring the rare signals discussed previously. To make the most of RHIC luminosities, the sPHENIX detector covers $2 \pi$ in azimuth $\phi \pm 1$ units in rapidity $(\eta)$ and reads out data at a rate of $15 \mathrm{kHz}$. To efficiently 
resolve jets and their energies, sPHENIX has both hadronic and electromagnetic calorimeters. Efficient tracking of particles from 0.2 to $40 \mathrm{GeV} / \mathrm{c}$ in transverse momentum $\left(p_{\mathrm{T}}\right)$ is needed for jet fragmentation measurements. Heavy flavor jet identification requires precision vertexing with a distance of closest approach in the x-y plane $\left(D C A_{\mathrm{xy}}\right)$ resolution of better than $70 \mu \mathrm{m}$. To measure the three $\Upsilon$ states in the dielectron decay channel, hadron rejection of better than $99 \%$ and a mass resolution of $1 \%$ at the $\Upsilon$ are required. These precise and efficient tracking requirements are met using a $1.5 \mathrm{~T}$ magnet and three tracking subsystems: a time projection chamber (TPC), a silicon strip intermediate tracker (INTT) and a precision MVTX detector based on ALICE's ITS upgrade.

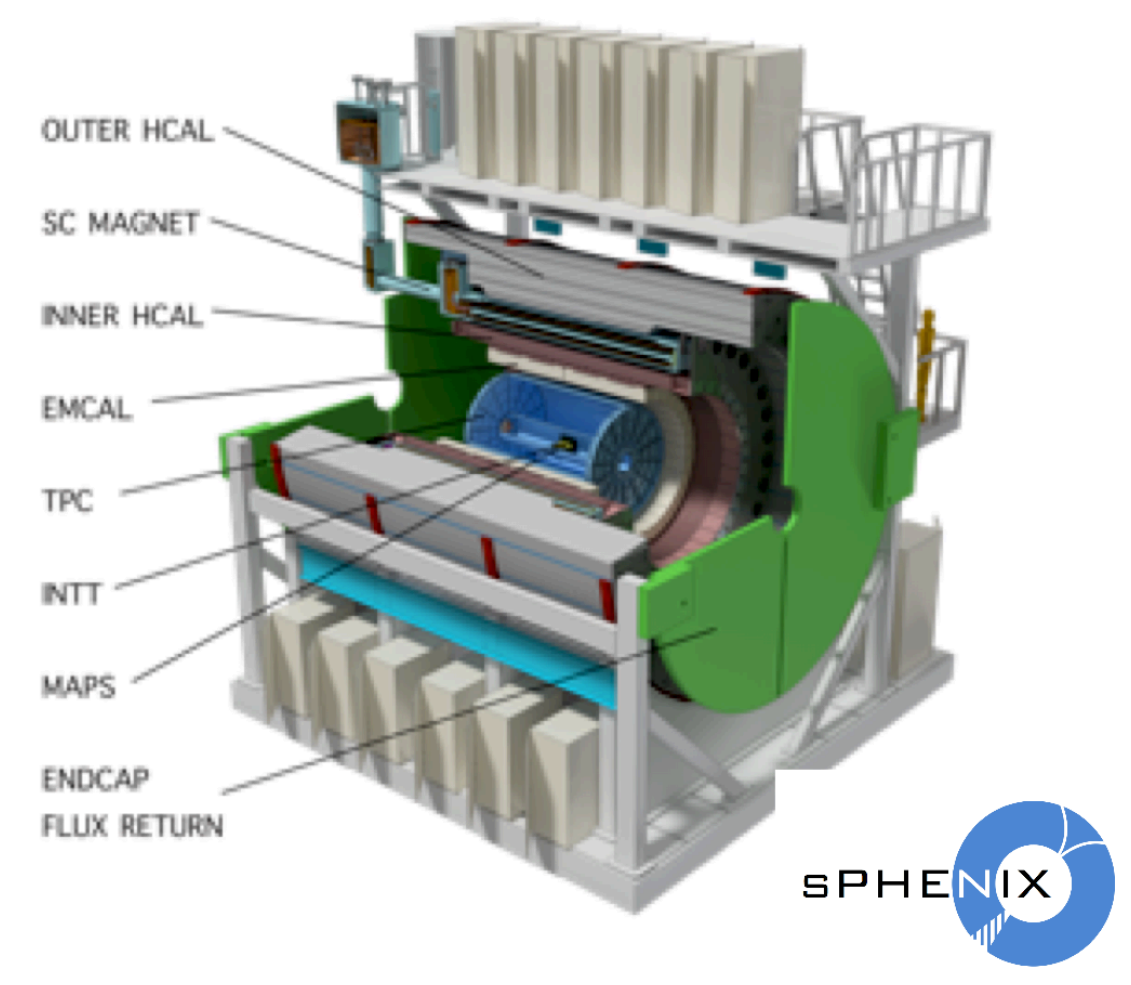

Figure 2: sPHENIX detector complex covering full azimuthal angle and rapidity range of $-1<\eta<1[$ []].

\subsection{Medium Energy Nuclear Physics with sPHENIX}

sPHENIX is planed to accumulate the integrated luminosity of $267 \mathrm{pb}^{-1}$ in $\mathrm{p}+\mathrm{p}$ collision in 2023 - 2025 running period. Following studies are made for the scenario of possible extension of sPHENIX running period beyond year 2025 and accumulate up to $700 \mathrm{pb}^{-1}$ with $60 \%$ doublelongitudinal polarization in $\mathrm{p}+\mathrm{p}$ collision at $\sqrt{s}=200 \mathrm{GeV}$. Measuring the gluon polarization $\Delta G$ has been one of the main goals of RHIC spin program and it has been measured with various probes. It has been reported [B] that the gluons inside a proton are positively polarized as much as $\Delta G$ of $0.2_{-0.07}^{+0.06}$ at $90 \%$ C.L. where $\Delta G$ is the integral of helicity gluon distribution function $\Delta g\left(x, Q^{2}=10 \mathrm{GeV}^{2}\right)$ in the region $x>0.05$. Key probes to determine $\Delta G$ at RHIC have been the double helicity asymmetries $A_{\mathrm{LL}}$ of inclusive jet measurements by STAR and $\pi^{0}$ measurements by 
PHENIX in central rapidity region at the collision energies $\sqrt{s}$ of 200 and $510 \mathrm{GeV}$. Thanks to the high hermeticity of sPHENIX and its high DAQ rate capabilities up to $15 \mathrm{kHz}$, it is expected to improve the precision of double helicity asymmetry $A_{\mathrm{LL}}$ measurements using jet as a probe by factor of $>4$ in the momentum fraction region $0.05<x<0.4$ [可].

On the other hand, direct photon production has been considered as a "golden" probe for $\Delta G$ measurement because the main contribution process in RHIC kinematics is gluon Compton scattering $(q g \rightarrow q \gamma)$. This sub-process is relatively simple compared to the mixed sub-process of jet's and $\pi^{0}$ 's $(g g, g q$, and $q q)$. The simplicity of the sub-process would laeve less room for the model dependence to interpret the data in the global next-to-leading-order (NLO) perturbative QCD fit process to extract $\Delta g(x)$. The main challenge for direct photon reconstruction in the sPHENIX barrel EMCal is the separation between a single photon and two merged photons from a $\pi^{0}$ decay. With the EMCal granularity $\Delta \phi \times \Delta \eta=0.024 \times 0.024$, the two photon merging probability is $50 \%$ for $\pi^{0} p_{\mathrm{T}} \sim 8 \mathrm{GeV} / \mathrm{c}$. Thanks to the projective geometry of the EMCal, a transverse shower profile analysis can be done with high precision, which can further extend the range for identification of single photon clusters from merged clusters to about $12-15 \mathrm{GeV} / \mathrm{c}$. Resulting statistical precision is expected to be improved by factor of 10 compared to existing measurements. The projected statistical error bar of jet (left) and direct photon (right) $A_{\mathrm{LL}}$ using sPHENIX barrel acceptance are plotted in Figure [ $]$ in comparison to the results of the DSSV14 global fit[[5].
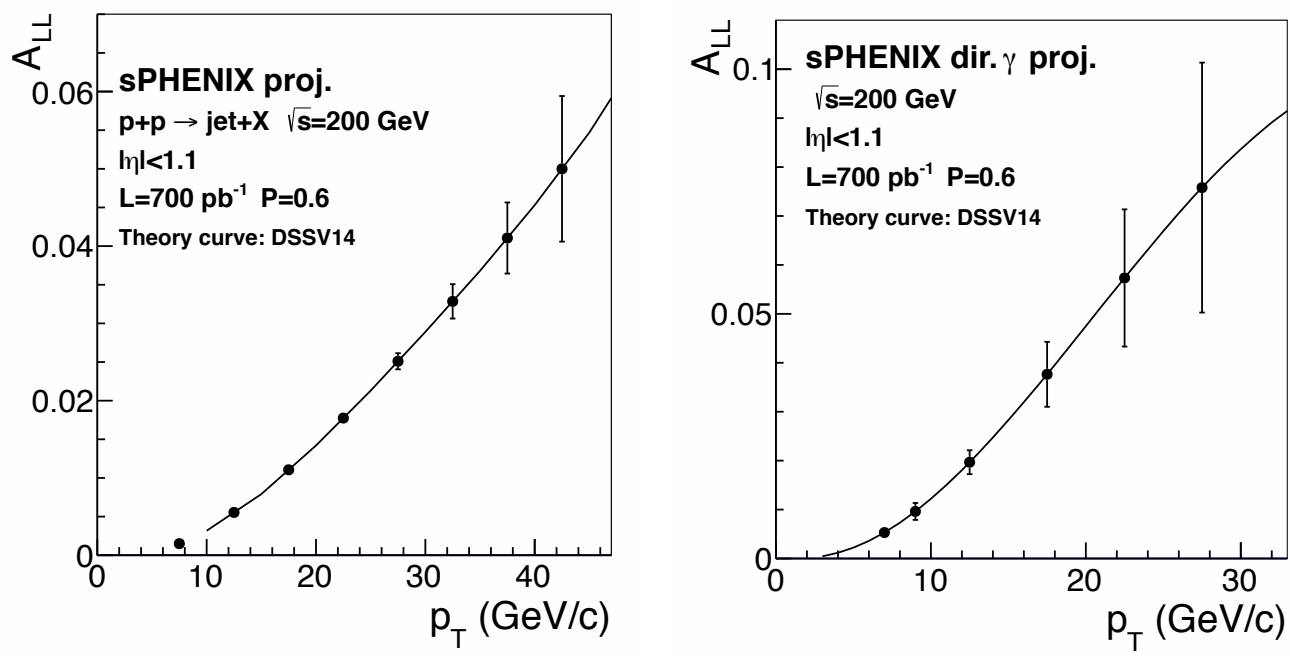

Figure 3: Projected statistical errors of jet (left) and direct- $\gamma$ (right) $A_{\mathrm{LL}}$ for assumed integrated luminosity of $700 \mathrm{pb}^{-1}$ in SPHENIX plotted on DSSV14 global fit curves[[]] as a function of $p_{\mathrm{T}}$.

\section{Forward Physics and Detector}

The fsPHENIX " forward sPHENIX" ) physics program [3] focuses on physics observables at forward angles with additional detectors augmenting the sPHENIX detector. With the possibility that in the transition to the EIC, fsPHENIX detector may be implemented during sPHENIX running period which adds the jet detection capability to the forward region as well. The fsPHENIX detector is thus designed to be compatible as an EIC day- 1 detector. The fsPHENIX physics program 
centers on the comprehensive set of measurements in transversely spin-polarized $p+p$ and $p+A$ collisions exploiting the unique capability of the RHIC collider to provide beams of protons with high polarization in addition to a variety of unpolarized nuclear beams. Transverse spin asymmetries (TSSA) from Collins and Sivers effects in jets and Drell Yan can be studied in the unexplored large Feynman momentum $x_{\mathrm{F}}$ region, where these effects are expected to be large. Large acceptance and fine momentum and angular resolution of the forward detector allows us to execute next generation measurements to disentangle these competing effects in TSSA.

The fsPHENIX will enrich not only the hadron structure studies, but also provide the opportunities to deeply investigate into the initial state of the QGP by covering a broad baryon density phase in heavy ion collisions. By expanding the rapidity coverage, a long rapidity range correlation of multi-particles allows access to the earlier stage of the QGP formation which is one of the critical studies to characterize the QGP. The angular correlations between pairs of rapidity-separated particles must have formed at early times of the QGP formation[ [0]. Shown in Figure $⿴ 囗 十$ is a space-time correlation diagram showing the causal relation between particles $a$ and $b$. If two correlated particles freeze out at a proper time $\tau_{\mathrm{F} . \mathrm{O}}$. then causality dictates that the correlation must have formed at an earlier proper time $\tau_{0}$ constrained by following relation:

$$
\tau_{0} \leq \tau_{\text {F.O. }} \exp \left(\frac{1}{2}\left|\eta_{a}-\eta_{b}\right|\right)
$$

where $\eta_{a}$ and $\eta_{b}$ are the momentum-space rapidity of particles $a$ and $b$, respectively (assuming the mass of the particles are negligibly small compared to their momentum).

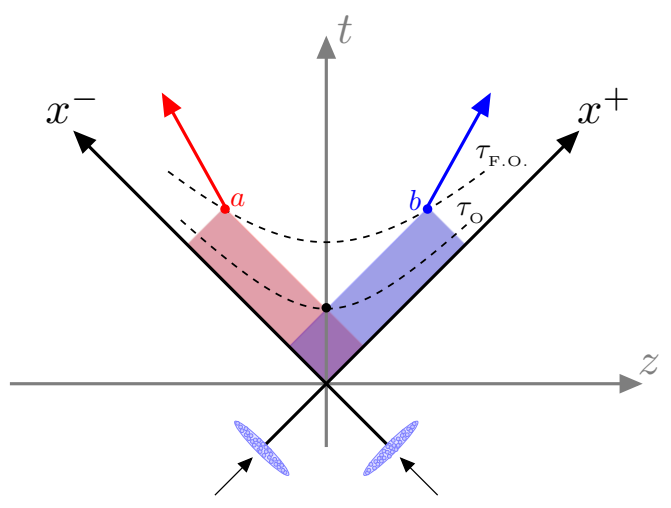

Figure 4: Space-time diagram showing the causal relation between particles a and b. Particle $a$ and $b$ are causally connected to the red and blue patches respectively. The correlation between them must have formed in their overlap at a proper-time before $\tau_{0}$.

\section{1 fsPHENIX Detector}

Shown in Figure [ is the sPHENIX detector with the conceptual forward instrumentation fsPHENIX in GEANT4[[]]. fsPHENIX covers the rapidity region of $1.4<\eta<4.0$. It is designed that tracking and electromagnetic calorimetry would be inside the magnetic field volume, while a hadronic calorimeter would be positioned outside the forward magnetic flux return. A magnetic field for particle track- ing and charge identification is provided by shaping the sPHENIX 
superconducting solenoid field with a high permeability piston located around the beam line in the forward region. Three new GEM stations at $z=150,200$ and $300 \mathrm{~cm}$ with a position resolution of $r d \phi=50-100 \mu \mathrm{m}$ would provide tracking and excellent momentum determination for charged particles $(\Delta p / p<0.3 \% p$ with $r d \phi=50 \mu \mathrm{m})$ over the full pseudorapidity range. An electromagnetic calorimeter will provide measurements of photons and electrons, while a hadronic calorimeter measures total jet energy, position and size. The hadronic calorimeter, with a jet energy resolution of $\Delta E / E 100 \% / \sqrt{E}$, follows the electromagnetic calorimeter and flux return.

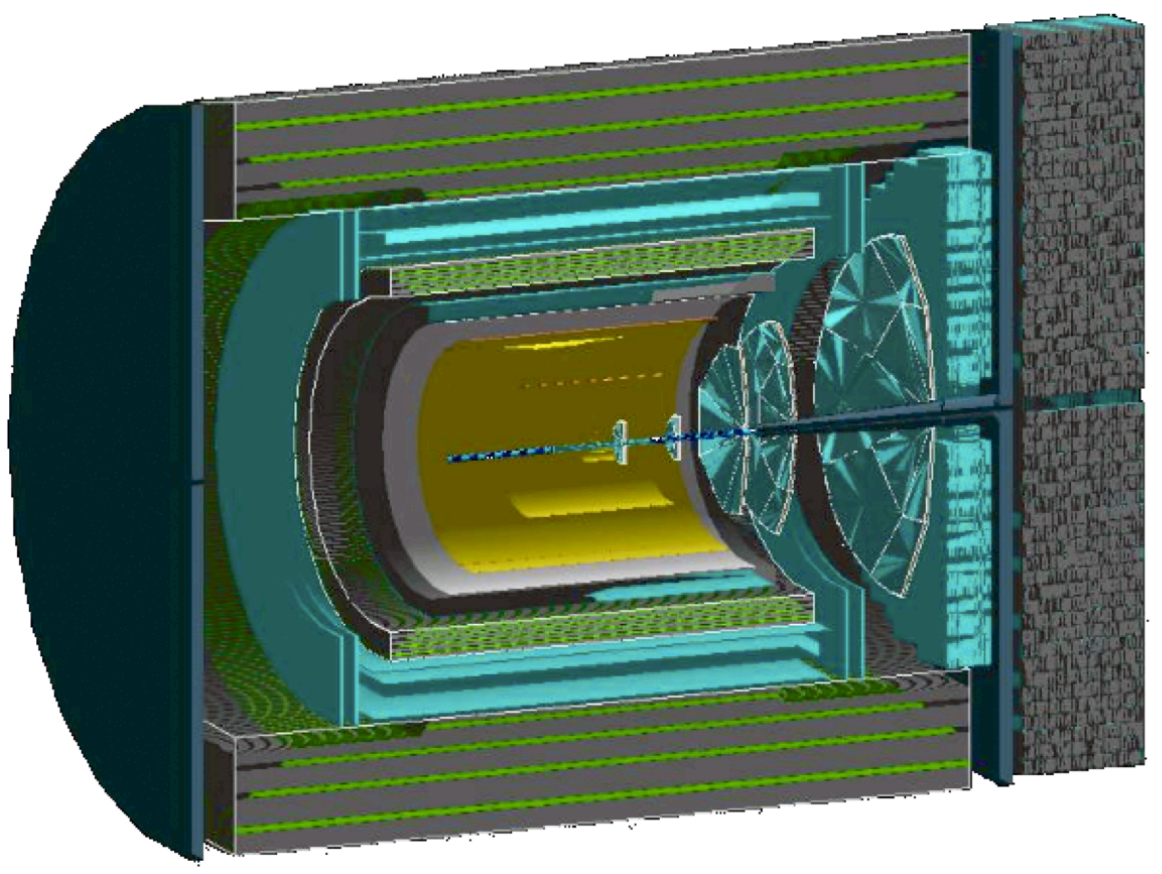

Figure 5: The sPHENIX detector with conceptual forward instrumentation fsPHENIX in GEANT4[[]]. The central tracker is approximated as a generic four-layer silicon-based tracking system with two small vertex GEM tracking stations in the forward direction.

\section{References}

[1] A. Adare et al.," An Upgrade Proposal from the PHENIX Collaboration, ” arXiv:1501.06197 [nucl-ex] (2015).

[2] A Letter of Intent "sPHENIX Forward Instrumentation", sPHENIX-note sPH-cQCD-2017-001 (2017).

[3] The RHIC Cold QCD Plan for 2017 to 2023: A Portal to the EIC, nucl-ex/arXiv:1602.03922 (2017).

[4] Medium-Energy Nuclear Physics Measurements with the sPHENIX Barrel, sPH-note sPH-cQCD-2017-002 (2017).

[5] D. de. Florian et al., Phys. Rev. Lett., 113, 012001 (2014).

[6] Kevin Dusling, Wei Li and Björn Schenke, International Journal of Modern Physics E Vol. 25, No. 01. 